\title{
RESIDUAL STRESS MEASUREMENTS IN CARBON STEEL
}

\author{
Joseph S. Heyman \\ National Aeronautics and Space Administration \\ Langley Research Center \\ Hampton, Virginia 23665 \\ Min Namkung \\ College of William and Mary \\ Williamsburg, Virginia 23185
}

External de magnetic field-induced changes in natural velocity of Rayleigh surface waves have been measured in steel specimens under varlous stress conditions. The low field slopes of curves representing the fractional changes of natural velocity have been proved to provide correct stress information in steels with different metallurgical properties. The slopes of curves under uniaxial compression, exceeding about one third of the yield stress, fall below zero in all the specimens when magnetized along the stress axis. The slopes under tension vary among different steels but remain positive in any circumstances. The stress effect is observed for both applied and residual stress. A physical interpretation of these results is given based on the stress-induced domain structure changes and the $\Delta E$-effect. Most importantly, it is found that the influence of detalled metallurgical properties causes only secondary effects on the obtained stress information.

\section{INTRODUCTION}

The practical application of the currently available techniques for nondestructive bulk residual stress measurements in metals is severely limited mainly due to a lack of calibration standard. This is because too of ten the stress effect is inhibited by material properties, such as texture, grain size, defect structure and so on. As a result, even the determination of the sign of the bulk residual stress can be made only under very specific conditions. Thus a technique, insensitive to material characteristics other than stress state and capable of differentiating the effects of tension from those of compression, is in great demand. In our previous papers (refs. 1 and 2) we reported a recent method measuring the uniaxial stress dependence of ultrasonic natural velocity changes induced by external dc magnetic field showing that compression can be detected unambiguously without knowing the zero stress standard.

The use of an external magnetic field in the ultrasonic velocity measurement In steel is based on two phenomena; the stress-induced domain structure change and the domain-dependent elastic modulus of a ferromagnet.

The unit cells of cubic crystals are not exactly cubic but slightly deformed in the ferromagnetic state (ref. 3). Specifically, the body-centered cubic (BCC) unit cells of iron below the Curie temperature are tetragonal with the longer 
edges parallel to the domain magnetization vectors oriented in the one of six equivalent directions of $\langle 100\rangle$. The effect of further deformation of unit cells on domains by uniaxial stress can be described by the magnetoelastic interaction. This interaction energy is lowest (highest) in domains oriented paralle1 (perpendicular) to the stress axis when an iron crystal is under tension and is opposite under compression. As in the case of magnetization with external magnetic field, the energetically favored domains expand their volume and a complete domain alignment (with zero net magnetization) can be achieved with sufficient uniaxial stress (ref. 4). The experimental verifications of such a stress-induced domain alignment have been made not only in pure iron but also in steel (refs. 5 and 6).

The elastic modulus of a nonlinear solid is defined as the ratio between infinitesimal applied stress and induced strain. In a ferromagnetic material the stress-induced strain, in general, includes the magnetoelastic strain as well as the direct elastic strain. The former is the strain induced by domain structure change with stress which aligns the longer edges of iron unit cells in certain directions with respect to the stress axis. The magnetoelastic strain is always positive under tension and is negative under compression for iron base alloys with BCC structure (ref. 7). Hence, the elastic modulus of a ferromagnet depends explicitly on 1ts domain structure. The contribution of magnetoelastic strain to the resultant elastic modulus can be separated by altering the domain structure with an external magnetic fleld. Such a domain-dependent elastic modulus of a ferromagnet is called the $\Delta E$-effect and is most pronounced in the domain wall motion region of the magnetization process (refs. 5 and 8 ).

The inftial domain structure is determined uniquely at each stress level. The subsequent change in domains due to external magnetic field will proceed differently according to the material stress. Therefore, the simultaneous changes In domain-dependent elastic modulus provides the stress information. Uniaxial stress affects the domain structure of iron only. by domain wall motion. Thus the stress information is expected in only the early stage of the magnetization process where domain wall motion occurs. This must be true in steels unless the crystalline anisotropy energy is drastically reduced by various impurities.

\section{ACOUSTIC MEASUREMENTS}

The dc magnetic field-induced ultrasonic wave natural velocity changes were measured using a pulsed-phase-locked-loop (P2L2) system described in detail elsewhere (ref. 9). A block diagram of this system is shown in figure 1. The basis of the measurement is a phase feedback scheme using a voltage-controlled oscillator $(\mathrm{VCO})$. The VCO output is gated to produce a tone burst of several cycles to drive a broadband transducer. The received pulse is amplified and phase detected using the VCO as a reference. A logic system samples the phase signal at the preselected point and causes the frequency of the VCO to change until quadrature is achieved. Once locked, the P2L2 maintains the quadrature condition with the change in frequency related to the change in sample properties as follows :

$$
\Delta \mathrm{F} / \mathrm{F}=\Delta \mathrm{V} / \mathrm{V}-\Delta \mathrm{L} / \mathrm{L}
$$




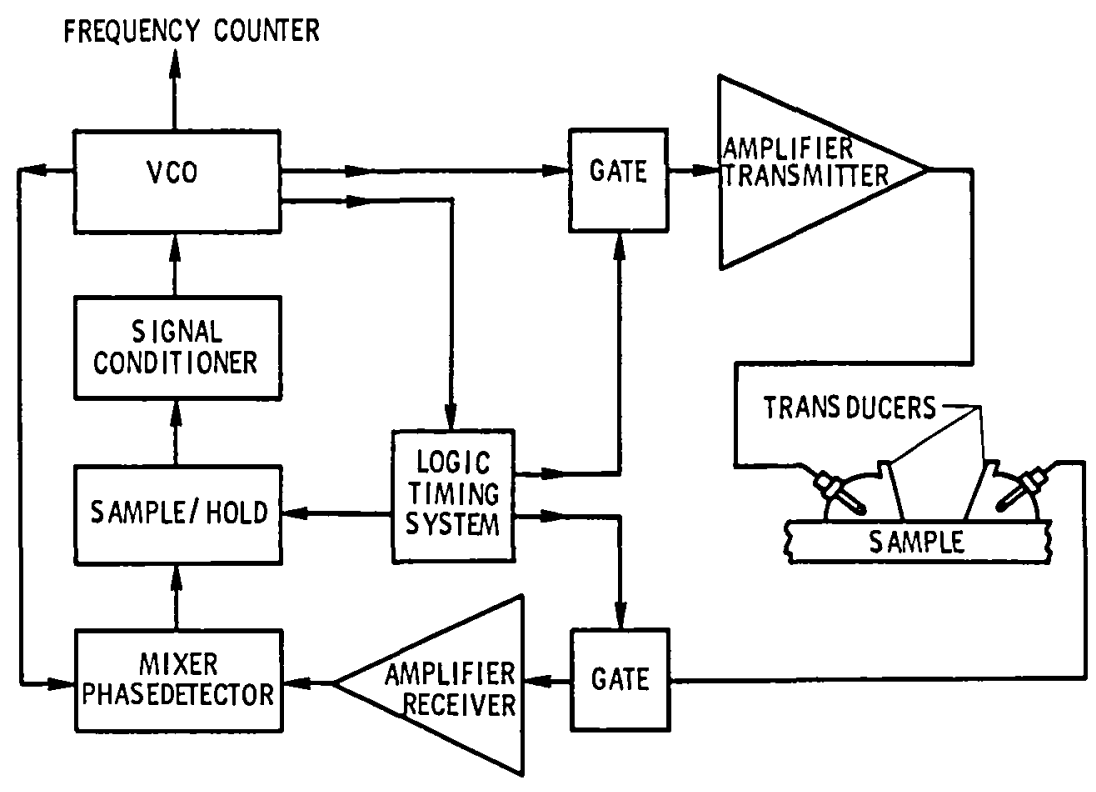

Figure 1. Block diagram of pulsed-phase-locked loop system.

where $V$ is the actual acoustic phase velocity and $L$ is the acoustic path length in the sample. $\Delta \mathrm{F} / \mathrm{F}$ is the fractional change in ultrasonic natural velocity. With the acoustic natural velocity, one does not have to measure the change in sample length during the measurement (refs. 10 and 11).

\section{PRELIMINARY EXPERIMENTS AND RESULTS}

Steel plates $(0.635 \mathrm{~cm} \times 5.08 \mathrm{~cm} \times 45.72 \mathrm{~cm})$ with unknown impurity composition were chosen to check the uniaxial stress effect on the domaindependent elastic modulus. Through this experiment surface waves propagated in the direction of the magnetic field which was parallel to the stress axis. A pair of longitudinal wave transducers, with a center frequency of $2.25 \mathrm{MHz}$, were mounted on variable angle blocks and used in the pitch-catch mode. Untaxial stress was applied to the specimen by four-point bending and the induced surface strain was measured with wire-type strain gauges. A major portion of the plate was magnetized by forming a closed magnetic circuit with a C-magnet. Each end surface of magnet was rounded to keep the contact area constant when the plate was bent. A Hall probe was inserted in a slit made on one of the C-magnet legs and the $\Delta F / F^{\prime} s$ were plotted against the measured field values.

To minimize any time-dependent effect, due to interstitial impurity migration or thermoelasticity, the following measurement procedure was employed. A sufficient relaxation time was allowed before the measurement at each stress level. The magnetic field was turned on and off while increasing the field strength to its maximum. The difference in the frequencies in the on and off states was divided by the off state value and taken as the $\Delta \mathrm{F}(\mathrm{H}) / \mathrm{F}$. After this, the specimen was demagnetized by a very low frequency ac field $(<\mathrm{Hz})$ with an exponentially decaying amplitude. 
The results are shown in figure 2 with the elastic surface micro-strain listed at the end of each curve. Here, the stress effect on $\Delta F(H) / F$ is clearly seen to lower its slope under compression and is opposite under tension. These measurements were repeated in many other specimens of the same material with the identical geometry. The position and the shape of curves varied somewhat from one specimen to another but the relative positions of curves and the stress effect were well reproducible.

The consistent effect of residual uniaxial stress on $\Delta F(H) / F$ was checked with permanently-bent plates (radius of curvature $=58 \mathrm{~cm}$ ) with the same initial geometry. It is well known from beam theory that the stretched side is under residual compression and the other side under residual tension when a plate is

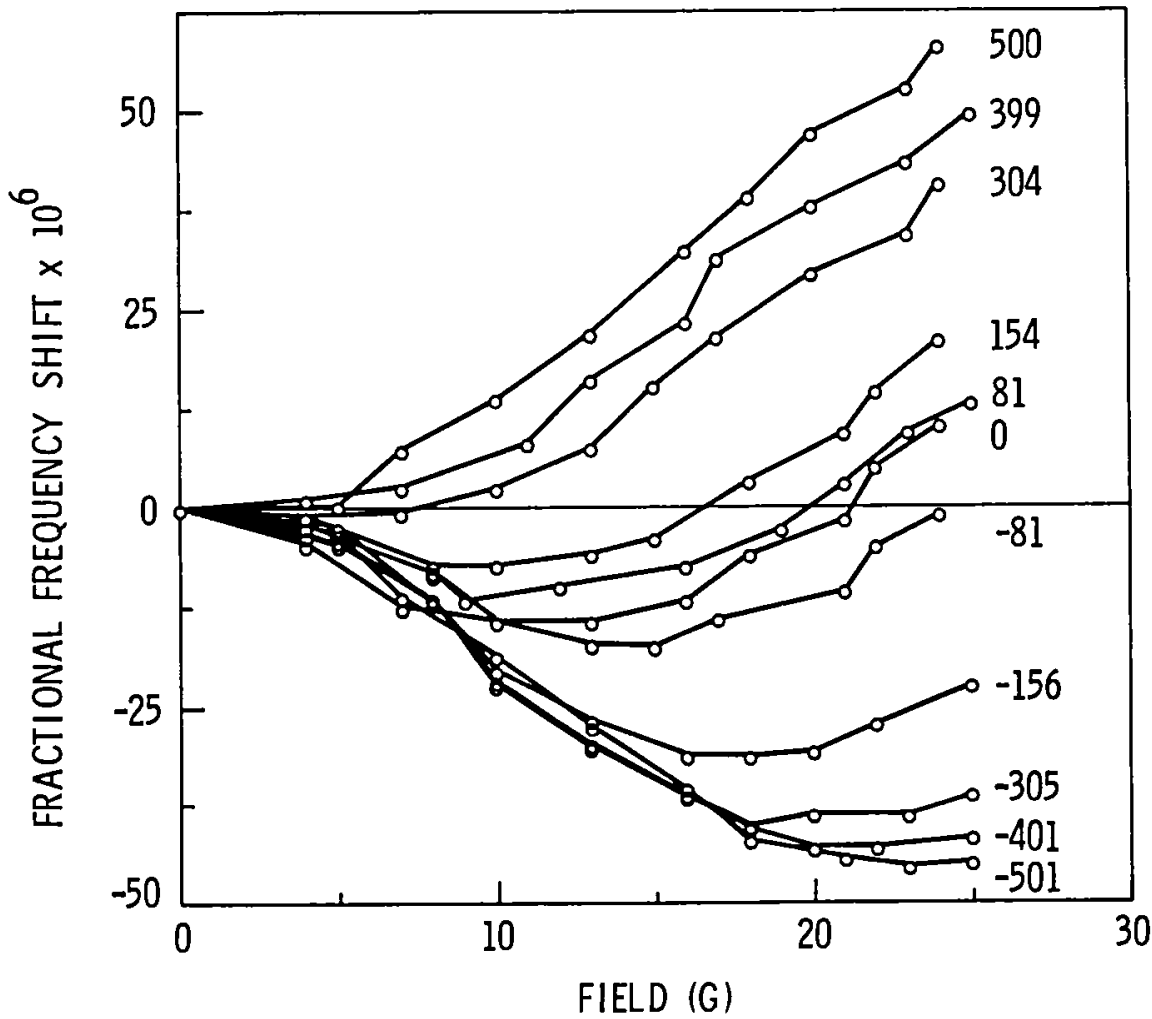

Figure 2. $\Lambda F(H) / F$ under applied tension and compression. Surface microstrain is labeled at each curve.

released after permanent bending with a finite radius of curvature. The results are shown in figure 3 for before and after bending. The curve labeled " $A$ " was taken in the surface stretched by bending and that labeled by " $B$ " was taken in the other surface. This result parallels the results for the applied stress state.

\section{MEASUREMENTS WITH CARBON STEELS AND RESULTS}

Steel samples with three carbon contents $(1020,1045$, and 1095) were used in this experiment. Two sets of samples were made for measurements with applied and residual uniaxial stress. 


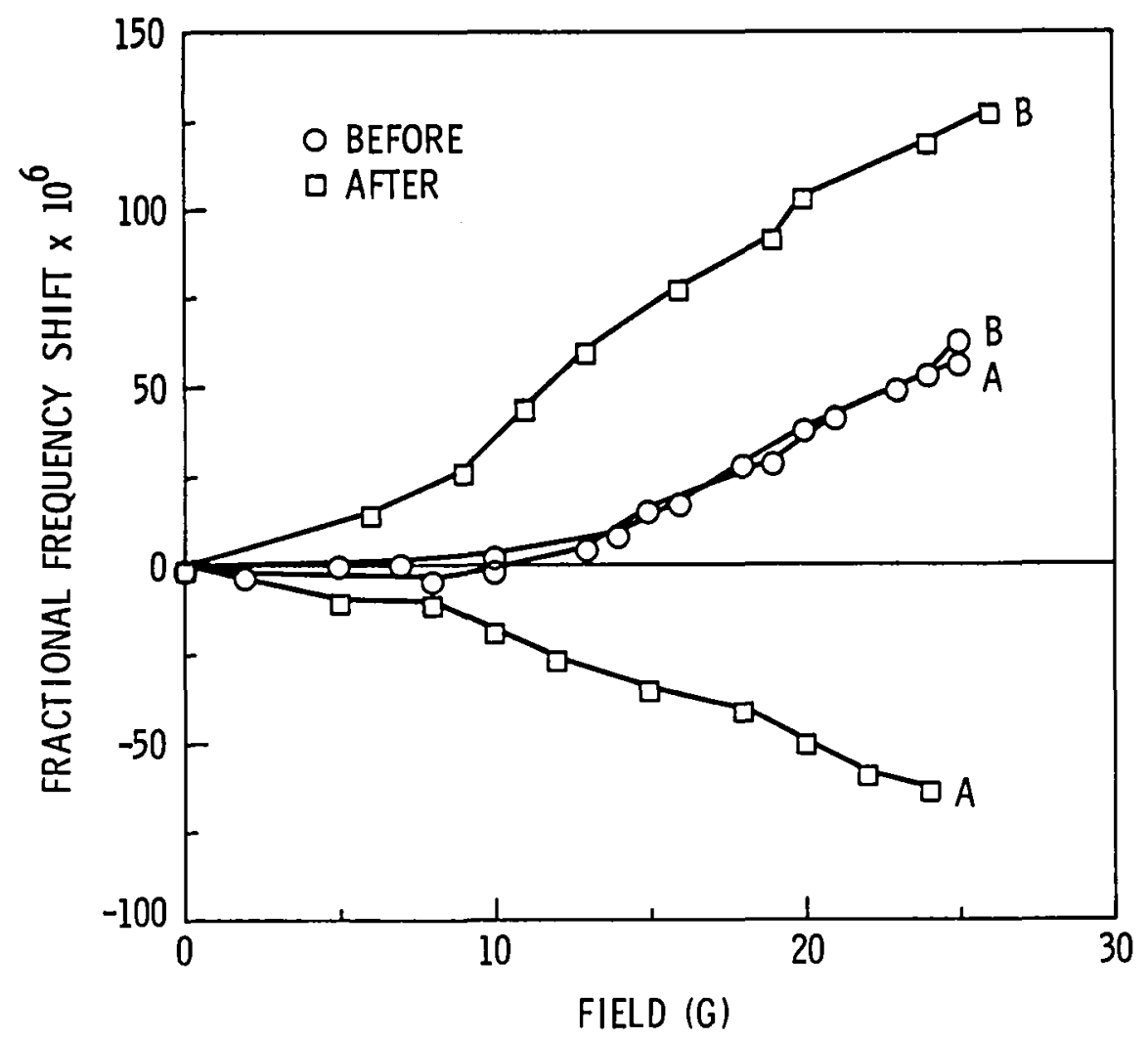

Figure 3. $\triangle \mathrm{F}(\mathrm{H}) / \mathrm{F}$ before and after permanent bending. For the square symbols, the label " $A$ " indicates the compressive surface and " $B$ " Indicates the tensile surface produced by permanent bending.

The samples were made from cylindrical rods of $2.54 \mathrm{~cm}$ in diameter and $30.3 \mathrm{~cm}$ in length. The center portion of these rods was machined to form a pair of parallel and flat surfaces $(1.8 \mathrm{~cm} \times 12 \mathrm{~cm})$. Both ends of each rod were threaded to fit into sample holders and placed in an INSTRON stress machine. Samples were magnetized lengthwise by a pair of water-cooled identical solenoids with C-shaped cores made of low carbon steel. Another set of samples were steel plates with the same carbon contents having dimensions of $3.8 \mathrm{~cm} \times 1.25 \mathrm{~cm} \times$ $30.3 \mathrm{~cm}$. Measurements were taken before and after permanently bending these plates with the radius of curvature $25.4 \mathrm{~cm}$. A specially designed C-magnet with rotatable arms was used to avold air gaps between the magnet ends and the curved surfaces. The total magnetic flux was measured by an integrating flux meter. The flux readings were callbrated with a big iron core magnet where the field Inhomogeneity was completely negligible for a region much larger than the dimension of the pickup colls.

The Raylelgh surface wave transducers with $5 \mathrm{MHz}$ and $2.25 \mathrm{MHz}$ center frequencies were used for rods and plates, respectively, in the pitch-catch mode. As before, ultrasonic waves propagated parallel to the field:applied in the stress axis. Measurements with external stress were taken in two different ways. First, the specimen was loaded to a desired stress level. Then, the magnetic field along the length of the specimen was monotonically increased to its maximum and decreased to zero while measuring $\Delta F / F$. At the end of this halfcycle measurement, the specimen was demagnetized. These measurements were 
repeated four or five times at each stress level. The previously mentioned discrete field on-off measurements were then made before stress was released. Such a sequence of measurement procedure was also used for the plates before and after permanent bending.

The continuous measurements exhibited a time dependence both when the external load was changed and in virgin specimens without load. The former was more pronounced and affected up to the first three measurements at each load level (each measurement took about 6 minutes) depending on the stress amplitude. This time dependence was caused by the combination of mechnical after effect (stressinduced directional ordering of elastic dipoles) (ref. 12), the magnetic after effect (mutual influence between ferromagnetic state and interstitial impurities) (ref. 13) and possibly the thermoelastic effect. The discrete measurements, on the other hand, gave almost time-independent results under any circumstances and the reproductbility was excellent. Hence, again the results with the discrete measurements will be presented here.

The results of $\triangle F / F$ in this section are plotted against the magnetic induction rather than internal magnetic field which is difficult to measure directly. Figures 4 and 5 show $\Delta F(B) / F$ in 1020 steel under external uniaxial

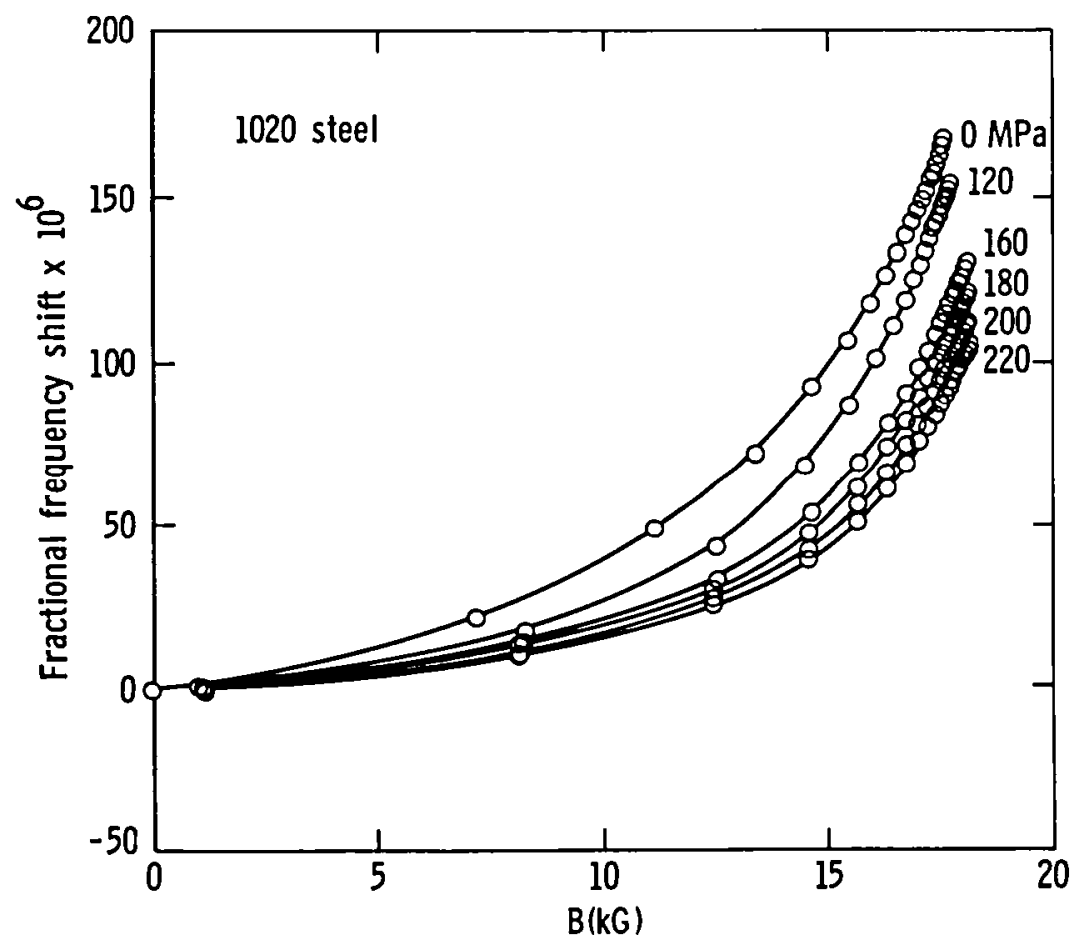

Figure 4. $\Delta F(B) / F$ in 1020 steel under applied tension.

tension and compression, respectively. Curves under high tension and those under low compression overlap. These curves move downward under tension but up to $220 \mathrm{MPa}$ the shape of curves remain very similar to that of curve without stress. Under compression, curves also move downward but, with increasing compression amplitude, their shape changes distinctively. At $-120 \mathrm{MPa}$ the negative initial slope begins to appear and 1 t becomes steeper under higher compression. 


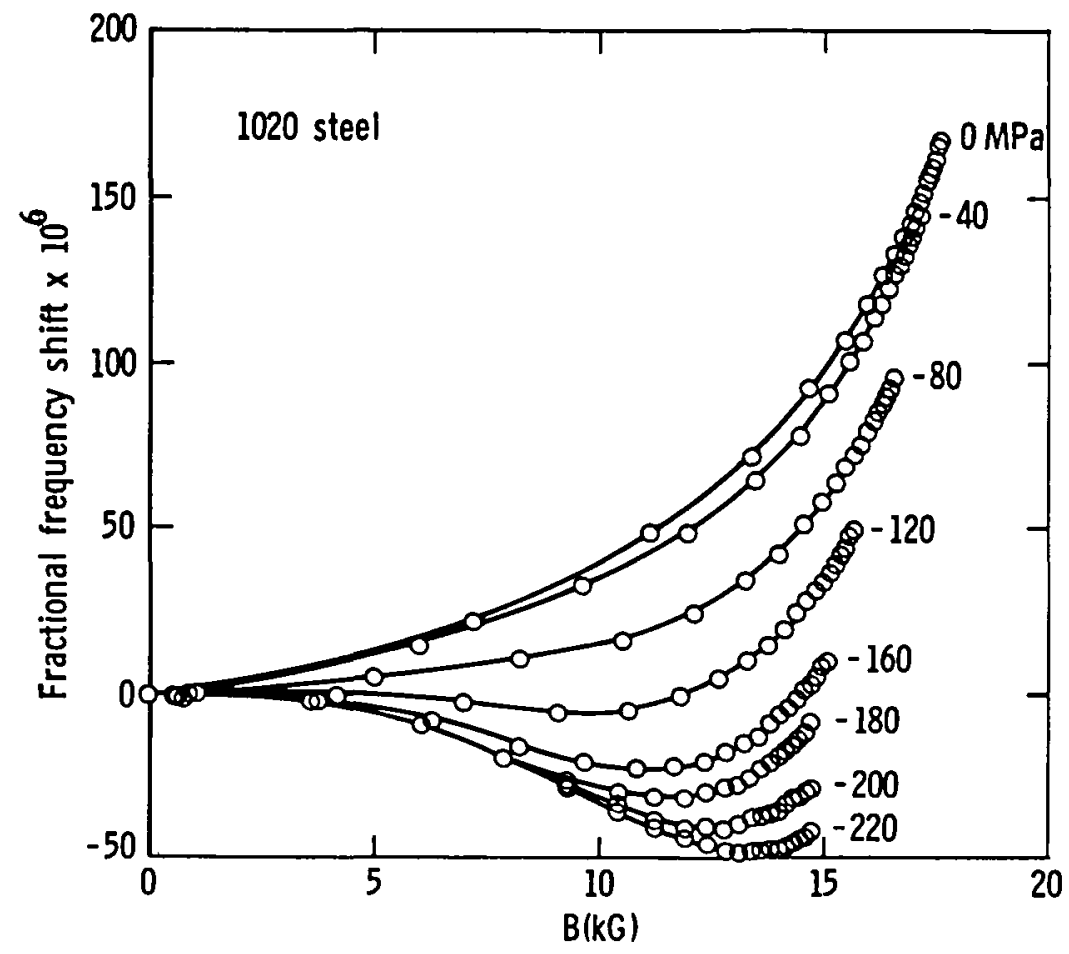

Figure 5. $\Delta \mathrm{F}(\mathrm{B}) / \mathrm{F}$ in 1020 steel under applied compression.

The results with 1045 rod are shown in figure 6. The tension curves in this specimen are distributed narrowly and all curves under $220 \mathrm{MPa}$ lie between the zero and maximum stress curves. Compression curves slightly overlap with tension curves and they spread downward with increasing compression magnitude. Here again, the negative initial slope begins to appear at $-120 \mathrm{MPa}$. Figure 7 shows the results with a 1095 rod. These results are very similar to those in the previous figures except that the negative initial slope of $\triangle F(B) / F$ begins to appear at a slightly higher value of compression.

The effect of tension on $\Delta F(B) / F$ has been seen to vary among different types of steels. This, as will be discussed later, depends on many metallurgical and structural properties of materials. The most important points are, however, the initial slope of $\Delta F(B) / F$ remains positive under tension and it only becomes negative under sufficient compression.

The consistent effect of residual stress was checked with permanently-bent plates with the same carbon contents. The measurements with the same plates before bending were made with an air core Helmholtz pair which was not able to magnetize properly the bent plates. Hence, the C-magnet with rotatable arms was used with the bent plates. Since total flux measured with the Helmholtz pair is different from the total flux in the plates only, measurements were repeated with other plates with the same carbon contents using the C-magnet. 


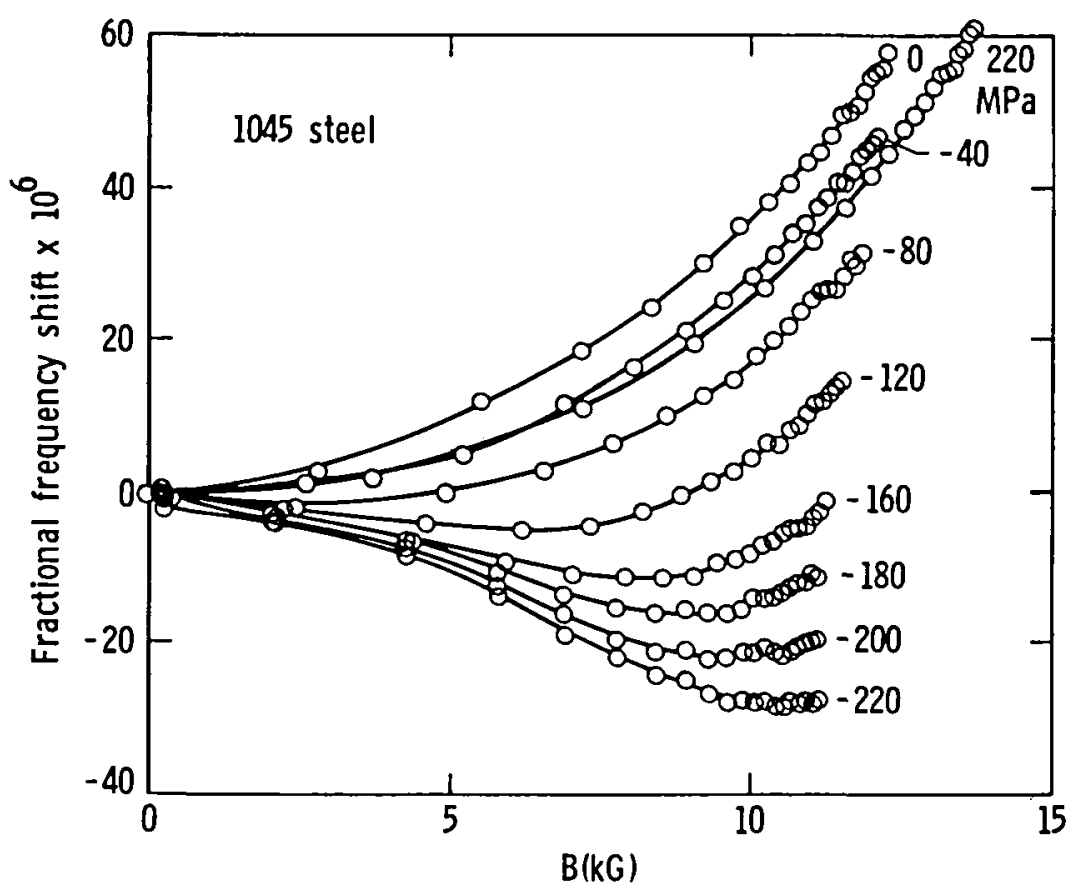

Figure 6. $\Delta F(B) / F$ in 1045 steel under applied tension and compression.

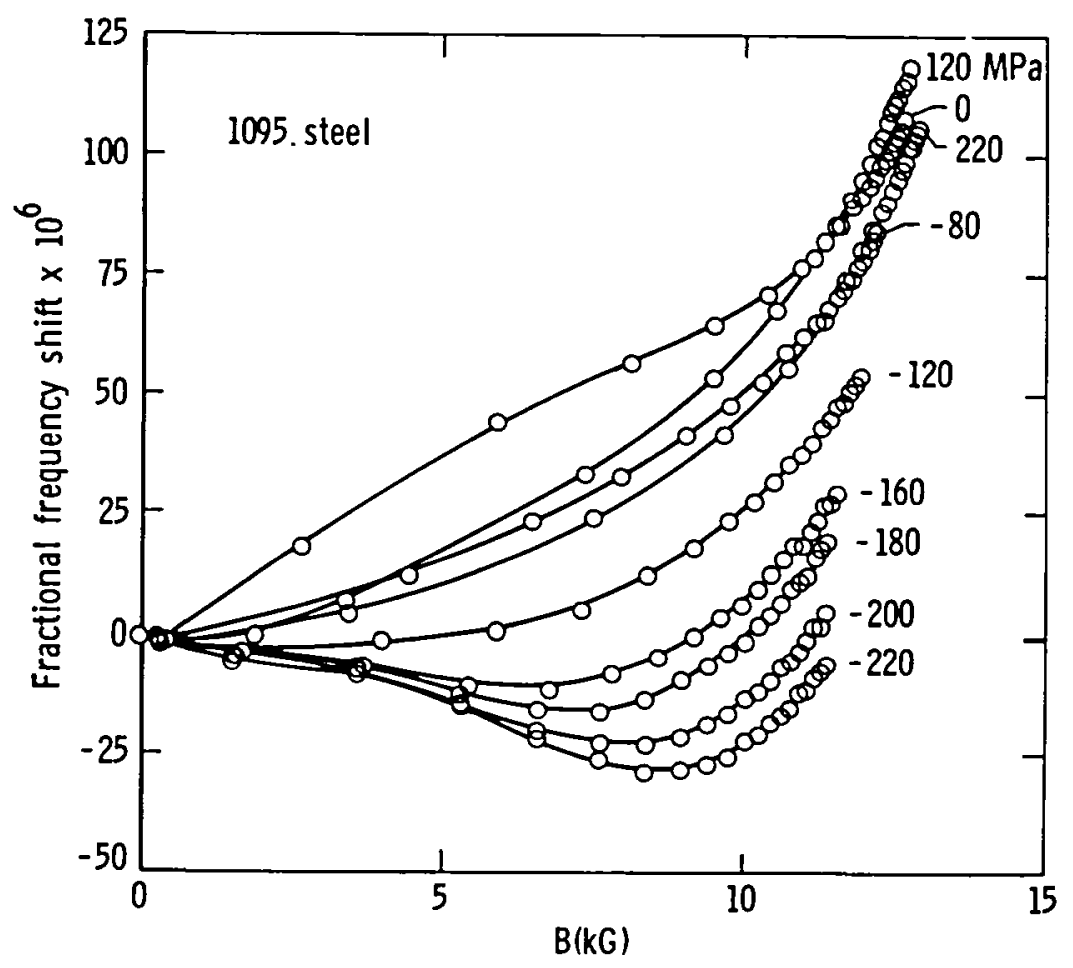

Figure 7. $\triangle F(B) / F$ in 1095 steel under applied tension and compression. 


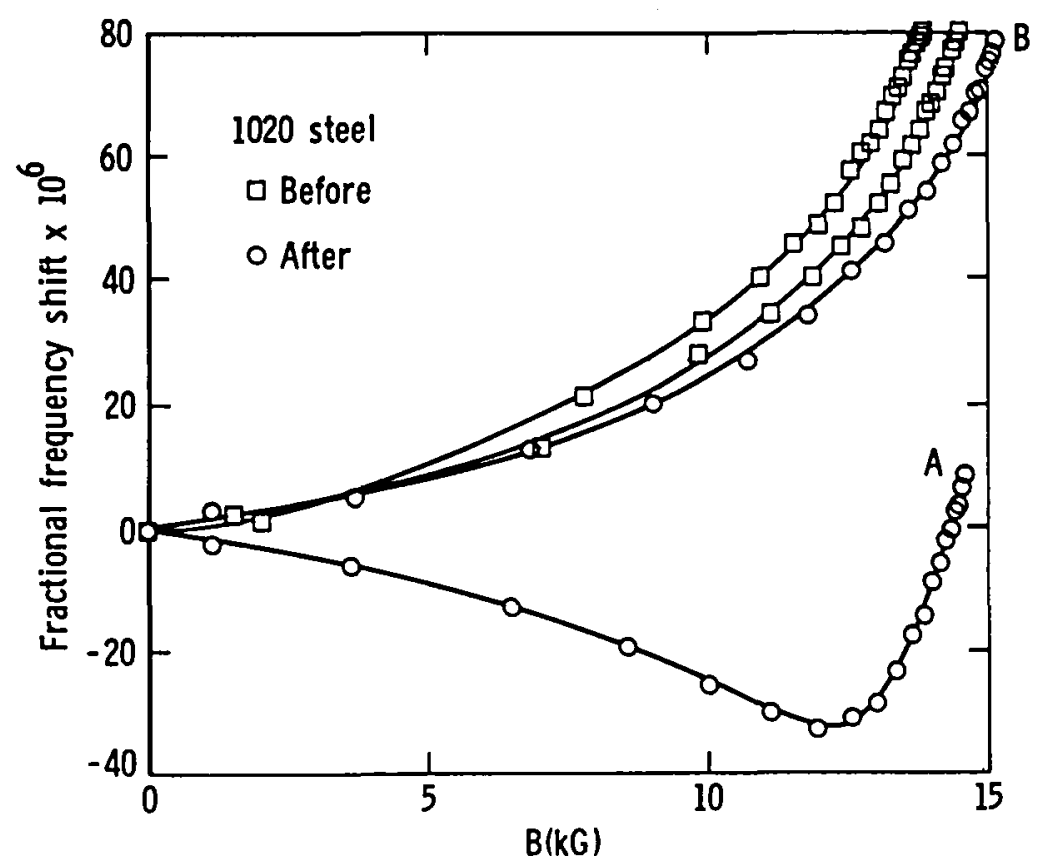

Figure 8. $\triangle F(B) / F$ in 1020 steel before and after permanent bending.

These results are shown in the next three figures for unbent and permanentlybent plates with the radius of curvature of $25.4 \mathrm{~cm}$. The stress effects on $\triangle F(B) / F$ generally follow what is expected from the results obtained with externally-applied uniaxial stress. In the 1045 plate the compression curve is almost a straight line passing through zero in the vertical axis indicating a very weak magnetization in that surface region. This is because magnetization depends on the stress state at each point in the material and it can be far lower in the surface under compression than that averaged over the sample cross section (ref. 7).

\section{DISCUSSION}

The presence of domain magnetization causes anisotropy of ultrasonic wave propagation in a crystalline solid (ref. 14). This is because the

ferromagnetic state is always accompanied by the spontaneous distortion of unit cells uniquely determined by the orientation of magnetization vector. This means that the wave velocity differs in different magnetic domains and in individual grains of a polycrystalline material. Hence, studies on the magnetoacoustic interaction have been limited to single crystalline materials beyond technical saturation. This certainly is not the present situation where we are interested in the magnetoacoustic phenomena during the domain wall motion only. For a qualitative discussion, however, the stress effect on $\Delta F(B) / F$ can be treated almost trivially by considering the following facts:

1. Uniaxial stress changes the domain structure in iron, and also in steel, only by domain wall motion. 


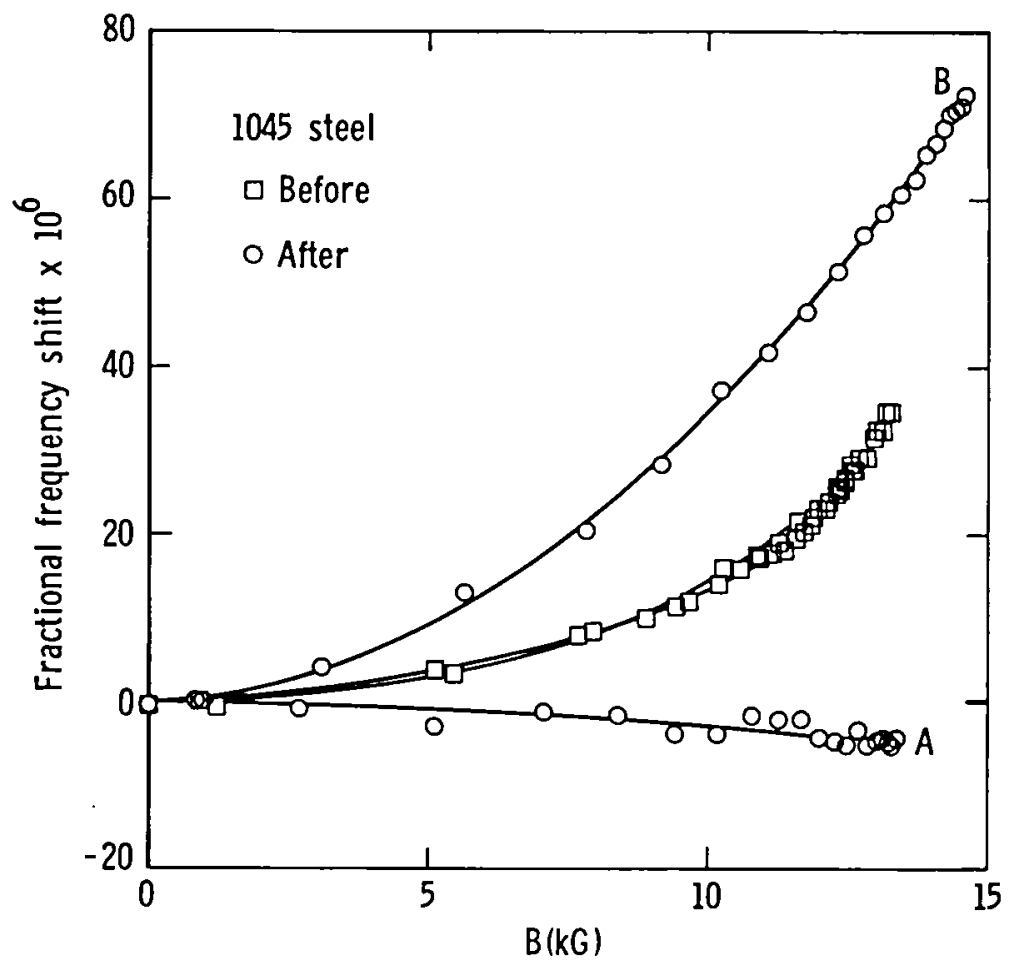

Figure 9. $\Delta F(B) / F$ in 1045 steel before and after permanent bending.

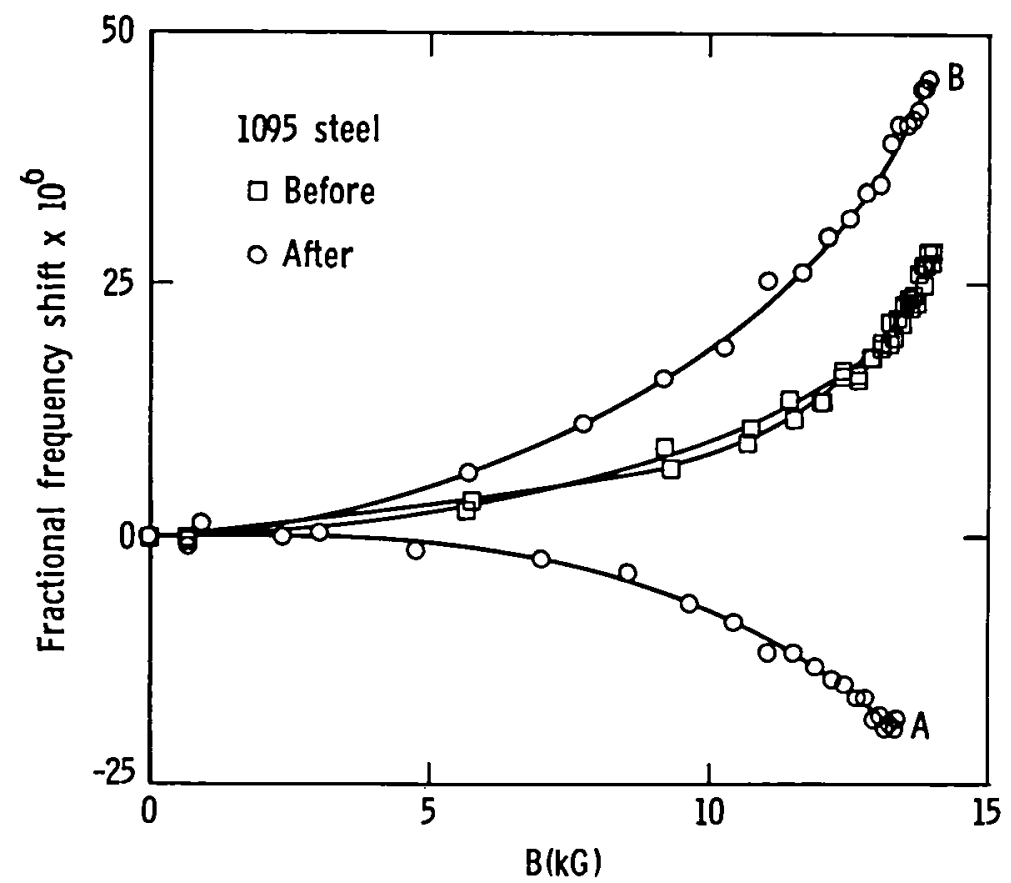

Figure 10. $\Delta F(B) / F$ in 1095 steel before and after permanent bending. 
2. The portion of $\Delta F(B) / F$ curves giving the stress information is solely contributed by field-induced domain wall motion.

Therefore, the discussion will focus on the slope of $\triangle F(B) / F$ with all the domains oriented in the easy axes of iron which are the six equivalent directions of $\langle 100\rangle$.

The mathematical description of magnetoelastic interaction gives the energy density in a domain subjected to uniaxial stress. This energy density is thermodynamically equivalent to a hydrostatic pressure acting on the domain boundary. The domain wall separating two domains feels a net pressure which is the difference in the magnetoelastic energy densities in the neighboring domains (ref. 15). As a result, the energetically-favored domains expand their volume in expense of less favored nelghboring ones by moving the domain wall. Among the two types of domaln walls in iron or steel, only 90-degree walls are moved by uniaxial stress while the positions of 180-degree walls are unaffected. Such a stressinduced 90-degree wall motion is resisted by various lattice imperfections (refs. 16 and 17 ).

Under sufficient tension, the majority of domain walls are those 180-degree walls close to the stress axis with a fraction of residual 90-degree walls remaining in the material. As the fleld is applied in the stress axis, the domains oriented close to the field axis expand their volume by moving both 180and 90-degree walls. The mobility of 180-degree walls is much higher than that of 90-degree walls not only because of the higher Zeeman energy difference across the walls but also the interaction with lattice imperfections is usually much less for 180-degree walls (ref. 18). Under sufficient compression, domains align perpendicular to the stress axis and the majority of walls are 180-degree walls. With the fleld applied in the stress axis, there is no Zeeman energy difference across 180-degree walls and only the residual 90-degree walls move. At the same time, the total area of 90-degree wall in the material grows. The area of 90-degree walls continues to grow until it reaches its maximum. After this point, as the magnetization proceeds, 90-degree walls begin to decrease and eventually vanish.

It has been already mentioned that the magnetoelastic strain is produced by aligning the longer side of iron unit cells into the applied tensile stress axis and is always positive. This, of course, is possible only by moving 90-degree domain walls. Hence, it is apparent that the presence of 90-degree walls effectively lowers the elastic modulus of the material.

The appearance of the negative slope of $\triangle F(B) / F$ in the early stage of magnetization along the uniaxial compressive stress axis is, therefore, well explained on the basis of initial domain alignment and the state of 90-degree walls. These, however, imply that curves under tension should be lower than that without stress because of less 90-degree walls to be removed by the field. This was seen at every stress level in 1020 and under high stress in other steels. The degree of stress-induced domain alignment, above al1, depends on many different material characteristics, such as saturation magnetization, spontaneous magnetostriction, local lattice strain and stress amplitude. There is a possibility that if the stress-induced domain alignment is difficult in a material then tensile stress merely helps the external field, in its own axis, remove 90-degree walls. Thus, the detailed shape of $\triangle F(B) / F$ curve depends on many different factors. The overall effect of sufficient compression causing the negative initial slope of $\Delta F(B) / F$, however, should be common in all materials of this type. 
In this paper, we presented clear experimental evidence of a technique capable of differentiating the effect of uniaxial compression from that of others both for applied and residual stresses. The Initial slope of natural velocity change in Rayleigh surface waves is negative under conpression and is positive otherwise when steel specimens are magnetized in the stress axis. The physical basis of this phenomena is provided by a simple model description. This is consistent with the results obtained in steels covering a fairly wide range of carbon contents. Hence, the effects of detailed material properties on the obtained stress information can be treated as secondary. 


\section{REFERENCES}

1. Namkung, M. and Heyman, J. S., Residual Stress Characterization with an Ultrasonic/Magnetic Technique, Nondest. Test. Comm., Vol. 1, 1984, pp. 175-186.

2. Namkung, M. and Heyman, J. S., Residual Stress Characterization with a Magnetic/Ultrasonic Technique, Proceedings of IEEE Ultrasonic Symposium, Nov. 1984, Dallas, Texas.

3. Kittel, C., Physical Theory of Ferromagnetic Domains, Rev. Mod. Phys., Vol. 21, 1949, pp. 541-584.

4. Namkung, M., A $\mu$ SR Study of Unlaxlal Stress Induced Symmetry Breaking in an Iron Single Crystal, Ph.D. Thesis, College of William and Mary, Oct. 1982.

5. Bozorth, R. M., Ferromagnetism, Van Nostrand, New York, 1951.

6. Dijkstra, L. J. and Martius, U. M., Domain Pattern in Silicon Iron Under Stress, U. M., Rev. Mod. Phys., Vol. 25, 1953, pp. 146-150.

7. Cullity, B. D., Introduction to Magnetic Materials, Addison-Wesley, Menlo Park, 1972.

8. Yamamoto, $M$. The $\Delta E$ Effect in Iron-Nickel Alloys, Sci. Rep., Tohoku Univ., Vo1. A11, 1955, pp. 102-119.

9. Heyman, J. S., Allison, S. G., and Salama, K., Influence of Carbon Content on Higher Order Ultrasonic Properties of Steel, Proceedings of IEEE Ultrasonic Symposium, Nov. 1983, Atlanta, Georgia.

10. Thurston, R. N. and Brugger, K., Third-Order Elastic Constants and the Velocity of Small Amplitude Elastic Waves in Homogeneously Stressed Media, Phys. Rev., Vol. 133, 1964, pp. Al604-1610.

11. Cantre11, J. H., Jr., Anharmonic Properties of Solids from Measurements of the Stress Acoustic Constant, J. Test. Eval., Vol. 10, No. 5, 1982, pp. 223-229.

12. Nowick, A. S. and Berry, B. S., Anelastic Relaxation in Solids, Academic Press, New York, 1972.

13. De Vries, G., Van Geest, D. V., Gersdorf, R., and Rathenau, G. W., Determination of the Magnetic Anisotropy Energy Caused by Interstitial Carbon or Nitrogen in Iron, Phisica, Vol. 25, 1959, pp. 1131-1138.

14. Alers, G. A., Neighbours, J. R., and Sato, H., Dependence of Sound Velocity and Attenuation on Magnetization Direction in Nickel at High Fields, J. Phys. Chem. Solids, Vol. 9, 1958, pp. 21-27.

15. Brown, W. F., Jr., Irreversible Magnetic Effects of Stress, Phys. Rev., Vo1. 75, 1949, pp. 147-154. 
16. Morrish, A. H., The Physical Principles of Magnetism, John Wiley and Sons, New York, 1965.

17. Craik, D. J., Structure and Properties of Magnetic Materials, Pion Limited, London, 1971.

18. Trauble, H., The Influence of Crystal Defects on Magnetization Processes in Ferromagnetic Single Crystals, in Magnetism and Metallury, Vol. 1, Edited by Berkowitz, A. E. and Kneller, E., Academic Press, New York, 1969. 